\title{
EL CONTROL POLICIAL EN LOS ALOJAMIENTOS DE USO TURISTICO Y SU INCIDENCIA EN LA SEGURIDAD
}

\author{
POLICE CONTROL IN THE CASE OF TOURIST \\ ACCOMMODATIONS AND ITS IMPACT ON SECURITY
}

\author{
ANA E. QUINTANA JIMÉNEZ ${ }^{1}$ \\ Doctora en Turismo por la Universidad Nebrija, Licenciada en Derecho por la \\ Universidad de Alcalá \\ Profesora de Derecho y de Turismo de la Facultad de Ciencias Sociales, \\ Departamento de Derecho, Universidad Nebrija.
}

\section{Resumen}

Los objetivos perseguidos a la hora de la realización de este trabajo han sido por un lado analizar si existe normativa específica que regule el control policial a través de los partes de entrada de viajeros en los alojamientos turísticos no reglados, en las Comunidades Autónomas con mayor presencia del Turismo en su PIB o que se han mostrado más activas en su regulación, determinando si esta falta de control afecta a la Seguridad de ese destino y en consecuencia produce un efecto negativo en el Turismo.

Para ello la metodología a utilizar será la jurídico-descriptiva, analizando las normas que regulan dicha modalidad, recurriendo a las fuentes primarias, y a la doctrina.

Como principales resultados cabría destacar que si bien recientemente se ha regulado la obligatoriedad de que dichos establecimientos turísticos estén en posesión de un libro registro donde archiven todos los partes de entrada de viajeros que se alojen en dicho inmueble, por CCAA, sin embargo, la Administración no dispone de instrumentos de control que permitan cuantificar el número de alojamientos y en consecuencia llevar a cabo su control.

El no poder realizar un control sobre el cumplimento de la obligatoriedad de comunicar a las Fuerzas y Cuerpos de Seguridad del Estado la identidad de los viajeros, comporta un riesgo para la seguridad de los destinos y aunque a día de hoy no genera todavía un efecto dañino sobre el turismo, puede terminar provocando una deslocalización hacia destinos más seguros. regulación

Palabras Clave: turismo, libro registro, economía colaborativa, alojamientos,

\section{Abstract}

The objectives pursued at the time of carrying out this work have been on the one hand to analyze whether there is specific legislation that regulates police control

1aquintan@nebrija.es 
through the entry parts of travelers in non-regulated tourist accommodation, in the Autonomous Communities with greater presence of Tourism in its GDP or that have been more active in its regulation, determining if this lack of control affects the safety of that destination and consequently produces a negative effect on Tourism.

For this the methodology to be used will be the legal-descriptive one, analyzing the rules that regulate this modality, resorting to the primary sources, and to the doctrine.

As main results it should be noted that although recently it has been regulated the obligation that said tourist establishments are in possession of a registry book where they archive all the entry parts of travelers staying in said property, by Autonomous Community, however the Administration does not It has control instruments that allow to quantify the number of accommodations and consequently carry out its control.

The not being able to carry out a control over the fulfillment of the obligatory nature of communicating the identity of the travelers to the State Security Forces and Bodies, entails a risk for the security of the destinations and although today does not generate a harmful effect yet on tourism, which may end up causing a relocation to safer destinations.

Key Words: Tourism, registry book, collaborative economy, tourist accommodation, regulation.

Fecha de recepción: 22 de marzo de 2019 Fecha de aprobación: 10 de junio de 2019

\section{INTRODUCCION}

La economía colaborativa es una de las tendencias que más está afectando a la transformación de todos los sectores, si bien es cierto que su incidencia ha sido especialmente relevante en el ámbito del turismo, y más específicamente en el transporte y en el alojamiento.

La economía colaborativa juega un papel importante en el turismo. En España la economía colaborativa supone entre el $1 \%$ y el $1,4 \%$ del PIB total ${ }^{2}$, pero la previsión es que para 2025 alcance el 2,9\%, lo que supondría una dinamización pasando de los 15.000 millones de ingresos que tuvo en 2015 a los 335.000 millones, 22 veces más, en la próxima década. ${ }^{3}$.

La repercusión social de la economía colaborativa en el turismo es tal, y es un fenómeno tan reciente, que es inevitable que suscite gran cantidad de incógnitas. El sector hotelero convencional se ha centrado en la competencia desleal que suponen

2 MODELOS DE NEGOCIO DISRUPTIVOS, Fundación Innovación Bankinter https://www.fundacionbankinter.org/documents/20183/156075/Modelos+Negocio+Disruptivos+Informe +2.pdf/e29a4961-1e52-4cfa-9de5-cc5576fab1d2.

3 ESTUDIO DE OSTELEA SCHOOL OF TOURISM \& HOSPITALITY, realizado en colaboración con EAE Business School, la Universidad de Lleida y la Rey Juan Carlos. https://www.tecnohotelnews.com/2018/04/economia-colaborativa-despegue-definitivo/. 
frente a sus hoteles, ya que estos alojamientos no $\operatorname{tributan}^{4}$, mientras que los establecimientos reglados no solo tributan, sino que además deben cumplir unos requerimientos para la obtención de licencias, avales bancarios, seguros, etc., en función de la modalidad de alojamiento a la que se refiera. Sin embargo, se obvia o se presta cuanto menos una menor atención a una diferencia fundamental, que afecta a un bien jurídico tan vital como la seguridad, que es la falta de control policial en los registros de clientes de este tipo de alojamientos. En este sentido, resulta bastante contradictorio, que la amenaza terrorista en la que estamos inmersos haya provocado un aumento de las medidas de seguridad en prácticamente todos los ámbitos, al tiempo que se relaja considerablemente en otros, como es el caso de este tipo de turismo. Especialmente relevante si se tiene en cuenta que una de las variables más influyentes en un turista a la hora de elegir un destino es la seguridad.

Nos encontramos, por tanto, con un porcentaje elevado de alojamientos turísticos no reglados que escapan al control policial, y en consecuencia suponen un problema para aplicar las medidas de control antiterrorista ${ }^{5}$.

\section{LA REGULACIÓN JURÍDICA DE LOS ALOJAMIENTOS DE USO TURISTICO}

\section{Español \\ 2.1 Los alojamientos de uso turístico en el Ordenamiento Jurídico}

El principal reto al que se enfrenta sector turístico es el control de la proliferación de las viviendas de uso turístico que debido a la implantación de plataformas web, como Airbnb, House Tryp, o Home Away, ha supuesto un cambio de escenario, en el que, por primera vez en la historia, las viviendas turísticas lideran el mercado turístico.

Según el V Barómetro sobre alquiler vacacional en España, realizado por la plataforma HomeAway, junto a la Universidad de Salamanca y Sondea (el Instituto de Investigación de Mercados a través de Internet), un 31,7\% de los españoles decidió alojarse en 2018 en una vivienda turística, un porcentaje que sube al 43,5\% en las preferencias de los turistas extranjeros.

El Ordenamiento Jurídico español no ha permanecido ajeno a esta nueva realidad y ha establecido su regulación en aspectos como la infraestructura, seguridad, sostenibilidad, confortabilidad y servicios.

Desde la perspectiva del Derecho Administrativo, antes de la trasposición al Ordenamiento Jurídico español de la Directiva 126/2006/CE, Directiva de servicios, conocida como Directiva Bolkestein ${ }^{6}$, era requisito obligatorio la solicitud de

\footnotetext{
${ }^{4}$ Se ha anunciado desde el Gobierno que se gravará a partir de 2019 con un 3\%, los servicios de plataformas digitales como Uber, Airbnb o Amazon para dar un "tratamiento fiscal adecuado" a las compañías que operan a través de Internet, especialmente las empresas de la llamada economía colaborativa, fundamentalmente de los sectores del alojamiento y el transporte.

${ }^{5}$ España se comprometió en virtud al artículo 45 del Convenio de aplicación del Acuerdo de Schengen, de 19 de junio de 1990, a adoptar las medidas necesarias para llevar a cabo las actividades de control sobre los viajeros que utilizaran establecimientos de hospedaje, por lo que el Estado Español está incurriendo en el incumplimiento del citado acuerdo.

${ }^{6}$ Directiva del Parlamento Europeo y del Consejo de la Unión Europea relativa a los servicios en el Mercado interno, presentada por la Comisión Europea en febrero de 2004 y aprobada finalmente en
} 
autorización administrativa siendo sustituido posteriormente por la inscripción del establecimiento en el correspondiente registro administrativo como requisito indispensable para poder iniciar su actividad. Dicha inscripción se realiza en el Registro de Empresas y Actividades Turísticas correspondiente de la Comunidad Autónoma a la que pertenezca. Por lo tanto, cualquier establecimiento turístico que se publicite o realice su actividad sin cumplir con los requisitos de inscripción en el registro preceptivo, se considerará un alojamiento de carácter ilegal, y se podrá aplicar la sanción correspondiente a la infracción cometida, que variará dependiendo de la Comunidad Autónoma, ya que las competencias en materia de turismo y su ordenación están transferidas.

Por otro lado si analizamos este tipo de alojamientos desde el punto de vista del Derecho Civil, la propia Ley 29/1994, de 24 de noviembre, de Arrendamientos Urbanos, sirvió a los propietarios de viviendas particulares como apoyo legal para su dedicación al sector turístico, a través de la figura del arrendamiento por temporada, recogido en su artículo $3.2^{7}$.

Esta utilización de la norma civil para no aplicar la turística, ha generado que la última modificación de la Ley de Arrendamientos Urbanos excluya expresamente de la misma el arrendamiento de carácter turístico.

\subsection{Normativa turística y seguridad}

Pero si hay un ámbito que justificaría la aplicación de la normativa turística es la relacionada con la seguridad, especialmente en el control del tráfico de visitantes.

La seguridad ciudadana es "la garantía de que los derechos y libertades reconocidos y amparados por las constituciones democráticas puedan ser ejercidos libremente por la ciudadanía y no meras declaraciones formales carentes de eficacia jurídica. En este sentido, la seguridad ciudadana se configura como uno de los elementos esenciales del Estado de Derecho" ${ }^{\text {. }}$

La seguridad para el Turismo ${ }^{9}$ se podría definir como, “Conjunto de medidas, condiciones objetivas y percepciones existentes en el ámbito social, económico o político de un destino turístico, que permiten que la actividad turística se desarrolle en un entorno de libertad, confianza y tranquilidad, y con la mayor protección física, legal o económica para los visitantes y sus bienes”.

Tanto desde el Ministerio del Interior como desde las Fuerzas y Cuerpos de Seguridad del Estado, se han propuesto una serie de medidas encauzadas para mejorar el nivel de seguridad en los turistas, se conoce como "Plan Turismo Seguro" ${ }^{10,}$, con el objetivo de incrementar la Seguridad Ciudadana en los destinos turísticos.

noviembre de 2006, entrando en vigor el 28 de diciembre de 2009 (Directiva 123/2006/CE), también se conoce como Directiva de Servicios.

7 Art. 3.2, “[...] tendrán esta consideración (arrendamiento para uso distinto de vivienda) los arrendamientos de fincas urbanas celebrados por temporada, sea ésta de verano o cualquier otra,[...]”.

${ }^{8}$ Tal y como parece recogido en el preámbulo de la Ley Orgánica 4/2015, de 30 de marzo, de protección de la seguridad ciudadana https://www.boe.es/buscar/pdf/2015/BOE-A-2015-3442consolidado.pdf

${ }^{9}$ SÁNCHEZ GÓMEZ- MERELO, www.belt.es 2015

${ }^{10}$ MINISTERIO DEL INTERIOR, Plan de turismo seguro. Disponible en: http://www.interior.gob.es/web/servicios-al-ciudadano/planes-de-prevencion/plan-turismo-seguro 
Un plan de seguridad en el Turismo debe contener preceptos encaminados a preservar la seguridad del turista, y mejorar sus niveles con respecto a la Seguridad ciudadana de los recursos turísticos, con el único objetivo de conseguir un entorno lo más seguro posible para que el turista se sienta protegido en todo momento.

Desde la Comisión Europea también se han hecho una serie de recomendaciones a los distintos Estados miembros dirigidas a ayudar a aplicar la normativa comunitaria en materia de "economía colaborativa" a través del documento denominado "Una Agenda Europea para la economía colaborativa"11 , de fecha 2 de junio de 2016, aunque no nos detendremos en él.

En el Plan Nacional Integral de Turismo (2012-2015) ${ }^{12}$, se recogía como uno de los objetivos "Impulsar la unidad de mercado: Desarrollar un marco normativo común que facilite e impulse el desarrollo del turismo en España, objetivo destinado a aproximar el amplio número de normativas existentes en los diferentes niveles territoriales (Estado, CC.AA. y Entes Locales.)”, y de esa forma impulsar la protección de la seguridad del turista y del residente, ya que al obligar al oferente del servicio a enviar un informe a la Dirección General de la Policía con los datos de registro del cliente, igual que en el resto de registros de alojamientos turísticos, se mejoraría la seguridad ciudadana.

La Ley Orgánica 4/2015, de 30 de marzo, de protección de la Seguridad ciudadana, artículo 25.1, BOE núm. 77, de 31 de marzo, establece que tanto "las personas físicas o jurídicas que ejerzan actividades relevantes para la seguridad ciudadana, como las de hospedaje, transporte de personas, acceso comercial a servicios telefónicos o telemáticos (...) quedarán sujetas a las obligaciones de registro documental e información, que deberán facilitar siempre que se le requiera, a los Cuerpos de Seguridad del Estado”.

Dicho parte estará en todo momento a disposición de los miembros de las Fuerzas y Cuerpos de Seguridad del Estado con competencia en la materia, quedando los establecimientos obligados a exhibirlo cuando a ello sean requeridos, y se deben conservar durante un plazo de tres años a contar desde la última fecha que lo integre.

Por tanto, hoteles, hostales, campings o bungalows deben enviar a la Guardia Civil y a la Policía Nacional datos personales como DNI o pasaporte, nombre, sexo, fecha de nacimiento, nacionalidad, fecha de entrada y salida de sus huéspedes antes de que pasen 24 horas de la entrada del viajero.

Se trata de unos formularios en los que deberán incluirse los datos, incluido DNI o pasaporte, de todas aquellas personas mayores de 16 años que se alojen en un establecimiento, con independencia de que sean españoles o extranjeros.

Actualmente, la Normativa básica reguladora de los alojamientos turísticos aplicable es la siguiente:

${ }^{11}$ La Comisión Europea ha presentado con fecha 2 de junio de 2016 las orientaciones a fin de que los consumidores, las empresas y las autoridades públicas puedan participar con confianza en la economía colaborativa. Estos nuevos modelos de negocio pueden aportar una importante contribución al crecimiento y el empleo en la Unión Europea si se fomentan y desarrollan de forma responsable.

12 SECRETARIA DE ESTADO DE TURISMO (SET) - Gobierno de España. Plan Nacional e Integral de Turismo, (PNIT) 2012-2015. SET. Madrid. Disponible en: http://www.minetad.gob.es/turismo/es-ES/PNIT/Paginas/que-es-PNIT.aspx 
Ley Orgánica 4/2015, de 30 de marzo, de protección de la Seguridad Ciudadana -artículo 25.1- (BOE núm. 77, de 31 de marzo), entró en vigor a raíz de los atentados yihadistas acaecidos en 2015.

Decreto 393/1974, de 7 de febrero, sobre identificación y registro de los usuarios de determinados establecimientos turísticos y de quienes alquilan vehículos con o sin conductor (BOE núm. 43, de 19 de febrero).

Decreto 1513/1959, de 18 de agosto, en relación con los documentos que deben llevar los establecimientos de hostelería referentes a la entrada de viajeros (BOE núm. 207, de 29 de agosto).

Orden INT/1922/2003, de 3 de julio, sobre libros-registro y partes de entrada de viajeros en establecimientos de hostelería y otros análogos (BOE núm. 165, de 11 de julio).

Orden INT/502/2003, de 21 de febrero, sobre creación de un fichero para el tratamiento de la información enviada por las instalaciones hoteleras a la Guardia Civil, en la prevención e investigación del terrorismo y de otras formas graves de delincuencia organizada, en la Dirección General de la Guardia Civil (BOE núm. 57, de 7 de marzo).

Resolución de 14 de julio de 2003, de la Secretaría de Estado de Seguridad, por la que se determinan las condiciones y forma de utilización de la transmisión de ficheros informáticos, así como las características de los soportes a que se refiere la Orden INT/1922/2003, de 3 de julio, sobre libros-registro y partes de entrada de viajeros en establecimientos de hostelería y otros análogos (BOE núm. 180, de 29 de julio).

\section{EL CONTROL DE LOS ALOJAMIENTOS DE USO TURÍSTICO EN ESPAÑA}

\subsection{Regulación del control en los alojamientos de uso turístico en España}

Como se observa, todas las regulaciones mencionan a la hotelería, solo encontramos una referencia a “otro tipo de alojamientos” en el Decreto 393/1974, de 7 de febrero, del Ministerio de la Gobernación sobre identificación y registro de los usuarios de determinados establecimientos turísticos y de quienes alquilen vehículos, con o sin conductor (BOE núm. 43, de 19 de febrero) ${ }^{13}$ :

Aunque dicho Real Decreto establece la necesidad de identificar a las personas alojadas en apartamentos no reglados y otros establecimientos de carácter turístico, dicho registro no se ha llevado a cabo en estos últimos, a diferencia de lo que ocurre en los hoteles, hostales y pensiones. La sanción impuesta a un alojamiento hotelero por un incumplimiento de estas características en la mayoría de Comunidades Autónomas tendría la consideración de una infracción leve, siempre y cuando no exista reiteración ni mala fe.

13 Decreto 393/1974, de 7 de febrero: "Para el conocimiento de los datos de identificación y tiempo de estancia de las personas alojadas en los establecimientos de hostelería, el Decreto 1513/1959 de 18 de agosto, estableció determinadas obligaciones que, se podrían aplicar a los apartamentos, bungalows, y otros establecimientos de carácter turístico, sin perjuicio de las competencias del Ministerio de Información y Turismo. Asimismo, resulta conveniente tener conocimiento de los datos personales de quienes alquilan vehículos de turismo, en determinados supuestos”. 
Por lo tanto, existe un importante número de alojamientos turísticos no reglados que están fuera del control policial, lo que dificulta la aplicación de las medidas de control antiterrorista, y en consecuencia el cumplimiento del artículo 45 del Convenio de aplicación del Acuerdo de Schengen, de 19 de junio de 1990.

\subsection{Libros registro: El parte de entrada de los viajeros}

Cualquier alojamiento turístico de hospedaje está obligado, por tanto, a llevar un libro-registro de control de entrada de viajeros, formado por los partes de entrada.

Los requisitos aparecen recogidos en la Orden INT/1922/2003 de 3 de julio, sobre libros-registro y partes de entrada de viajeros en establecimientos de hostelería y otros análogos. Es obligatorio el uso del modelo de parte de entrada recogido en el anexo de la propia Orden, ${ }^{14}$ aunque en el caso de algunos establecimientos se permite el uso de otros modelos siempre y cuando los Fuerzas y Cuerpos de Seguridad del Estado lo autoricen, por ejemplo, en el caso de la Policía Nacional con la aplicación informática Webpol ${ }^{15}$.

Los Cuerpos Policiales competentes en esta materia son:

- La Policía Nacional si en el municipio del alojamiento hay una comisaría de la Policía Nacional, se puedes utilizar la aplicación de la Policía Webpol. Hospederías $^{16}$.

La Guardia Civil en el resto de casos, se puede utilizar la aplicación de

Los Mossos d'esquadra en Cataluña, a través de la aplicación de Registro de Establecimientos de Hospedaje ${ }^{17}$.

Hotelero $^{18}$.

La Ertzaintza en el País Vasco, a través de su aplicación de Registro

Expresamente la mencionada Orden en su apartado segundo determina que:

1. Los impresos de partes de entrada de viajeros se adaptarán a lo recogido en su Anexo I, y también se permitirá realizar el registro a través de medios informáticos autorizados. $^{19}$

\section{${ }^{14}$ ANEXO I}

${ }^{15}$ CUERPO NACIONAL DE POLICÍA, aplicación informática para realizar los registros de los viajeros, para comenzar hay que hacer el alta en la aplicación llamada WebPol de la Policía, para ello es necesario llamar a la comisaría del distrito de su apartamento turístico y pedir cita.

${ }^{16}$ CHEKIN. Cómo hacer el registro de viajeros en hospederías (Guardia Civil) Disponible en: https://chekin.io/blog/como-hacer-el-registro-de-viajeros-en-hospederias-guardia-civil/

${ }^{17}$ GENERSALITAT DE CATALUNYA Registro de Turismo de Cataluña. Establecimientos hoteleros. Disponible en: https://web.gencat.cat/es/tramits/tramits-temes/Inscripcio-dEstablimentsHotelers-al-Registre-de-Turisme-de-Catalunya

18 ERTZAINTZA: Registro Hostelero. Disponible en: https://www.ertzaintza.eus/wps/portal/ertzaintza/!ut/p/z0/04_Sj9CPykssy0xPLMnMz0vMAfIjo8ziPd2MP ULD3AwMDMy8zQw8fdyNLEzNDL2Cgs31g1OL9QuyHRUBvvLPfQ!!/

${ }^{19}$ WEBPOL, E-HOTEL, CHEC-KIN 
2. Así también se establece que sea cual fuere la forma elegida para la elaboración del parte de entrada de viajeros, siempre deberá aparecer firmado por el cliente, tanto si se realiza manuscrita en papel, o a través de los medios informáticos creados a tal efecto como Webpol.

3. El establecimiento será el encargado de custodiar y archivar el parte de entrada que pasara a formar parte del libro-registro de dicho alojamiento, ordenándose de forma correlativa y constituyendo libros o cuadernos que integrarán un mínimo de 100 hojas y un máximo de 500. Se permite el archivo de libros en formato electrónico.

4. El libro-registro deberá estar en todo momento a disposición de los miembros de las Fuerzas y Cuerpos de Seguridad con competencia en la materia, quedando los propietarios del alojamiento obligados a exhibirlo cuando a ello sean requeridos.

5. Es importante tener en cuenta que los establecimientos deberán conservar los libros-registro durante el plazo de tres años.

No cumplir con estas este proceso de identificación en la policía puede acarrear sanciones que van desde los $600 €$ hasta los $30.000 €$, dependiendo si es una falta leve 0 grave, de conformidad con lo establecido en la Ley Orgánica 4/2015, de 30 de marzo, de Protección de la Seguridad Ciudadana.

\subsection{Análisis del registro de viajeros por Comunidades Autónomas}

La Constitución española en su artículo $148.1^{\circ} 18^{\circ}$ transfiere la competencia en ordenación y promoción del turismo a las Comunidades Autónomas en su ámbito territorial, por lo que cada Comunidad Autónoma podrá regular dichos alojamientos con una normativa propia, siempre y cuando se encuentre recogida dicha competencia en su Estatuto de Autonomía.

Debido a la variedad de normativa aplicable dependiendo de cada Comunidad Autónoma y a que se trata de una nueva modalidad de alojamiento, se ha decidido realizar un breve análisis por Comunidades, analizando solo aquellas en las que el turismo juegue un papel importante para su economía, o bien hayan sido pioneras o especialmente activas a la hora de responder jurídicamente a esta nueva realidad.

\subsubsection{ANDALUCÍA}

La importancia del Turismo en Andalucía es evidente. Tal y como se reconoce desde la Junta de Andalucía, la cifra anual de visitantes supera los 28,2 millones, más del triple de la población regional, y los ingresos alcanzan los 19.000 millones de euros. Andalucía es la comunidad autónoma más visitada por los turistas españoles y se sitúa entre los primeros destinos en cuanto a receptora de extranjeros, especialmente británicos, alemanes y franceses. Según los últimos datos disponibles, la región suma unas 460.000 plazas de alojamiento reglado, de las que 250.000 se ofertan en la tipología hotelera.

Debido a la importancia del Turismo en la región y la proliferación de este tipo de alojamientos turísticos, se modificó el Decreto 194/2010, de 20 de abril, de establecimientos de apartamentos turísticos, mediante el Decreto 28/2016, de 2 de febrero, de las viviendas con fines turísticos, de forma que en su artículo 7 se reconoce:

“Artículo 7. Formalización del contrato y acceso a la vivienda. 
2. Las personas usuarias, para hacer uso de la vivienda, deberán presentar su documento de identificación a los efectos de cumplimentar el correspondiente parte de entrada conforme a la normativa vigente sobre libros-registro".

En Andalucía por tanto, al disponer de una normativa de reciente entrada en vigor, si se ha tenido en cuenta esta modalidad, a efectos de regular su control. Como características esenciales del alquiler vacacional podemos citar las siguientes que constan en el Decreto 28/2016, de 2 febrero, de las viviendas con fines turísticos:

1.- Expulsión inmediata del inmueble: Art.2.5. Cuando las personas usuarias incumplan alguna de las obligaciones que establece la L 13/2011, de 23 de diciembre, especialmente las relativas a las reglas de convivencia, las personas o entidades explotadoras podrán denegar la permanencia de las personas usuarias y requerir el abandono de la vivienda, en el plazo de veinticuatro horas.

\subsubsection{ASTURIAS}

Asturias ha ido incrementando en los últimos años el número de pernoctaciones destacando el fuerte incremento de las viviendas vacacionales con un aumento del $22,8 \%$ en alojamientos y el $21,4 \%$ en plazas, mientras que la hotelería presenta una disminución del $0,3 \%$ en la oferta de plazas en las categorías medias y bajas, y un leve incremento en las más altas. Este panorama justifica la existencia de una norma que regule la oferta de viviendas privadas para el uso turístico.

En esta Comunidad es de aplicación el Decreto 48/2016, de 10 de agosto, de viviendas vacacionales y viviendas de uso turístico, en cuyo artículo 27 determina que además de la observancia de las obligaciones establecidas en el artículo 23 de la Ley 7/2001, de 22 de junio, de Turismo, las empresas explotadoras de las viviendas serán las responsables de controlar las entradas y salidas de los usuarios turísticos y de remitir a la Dirección General de la Policía la información relativa a la estancia de las personas que se alojen en dicho establecimiento.

También será de aplicación Ley 7/2001, de 22 de junio, de Turismo. En el Capítulo II dedicado a las empresas turísticas, en su artículo 23, se incluyen los preceptos donde se establecen las obligaciones de las empresas turísticas, aunque expresamente no se hace ninguna mención a la al traslado del parte de viajeros a las Fuerzas y Cuerpos de Seguridad del Estado, sí que se establece la obligatoriedad de contar con los libros y demás documentación que sean exigidos por la reglamentación vigente, pudiéndose considerar incluido, por tanto, el parte de entrada de viajeros ${ }^{20}$.

El Decreto 48/2016 también establece que las empresas explotadoras de las viviendas estarán obligadas a respetar los preceptos contenidos en la Ley 7/2001, de 22 de junio, de Turismo, las del Decreto 48/2016, así como las ordenanzas municipales y el resto de normas sectoriales aplicables, fundamentalmente las relativas a la seguridad, al urbanismo, a aspectos técnicos, y la accesibilidad.”

La duda de si las personas físicas están incluidas en el término "empresas turísticas" se aclara en el artículo 3, donde se define la empresa turística como las que "las personas físicas o jurídicas que, de manera habitual y con ánimo de lucro, realicen una actividad cuyo objeto sea la prestación de alguno de los servicios turísticos”.

${ }^{20}$ PARTEE, Normativa de partes de entrada de viajeros y libros-registro en alquiler turístico. Disponible en: https://partee.es/normativa-de-libros-registro-y-partes-de-entrada-de-viajeros/. 
Por tanto, en Asturias si existe una obligación expresa a dar traslado a las Fuerzas y Cuerpos de Seguridad del Estado de los partes de viajeros en los alojamientos turísticos y viviendas vacacionales.

\subsubsection{CANARIAS}

El inicio del Turismo en las Islas Canarias se remonta a los años 60, cuando el auge del turismo de sol y playa se adueñaba de la demanda. Actualmente dicho turismo sigue siendo clave para el destino, pero se ha producido una diversificación del producto turístico, incorporando el turismo cultural, rural o de congresos.

Durante el 2015, la actividad turística en Canarias obtuvo un resultado de 13.480 millones de euros, un dato que permitió elevar la contribución de este sector en la economía canaria hasta el 31,9\%, dinamizar de forma importante otros sectores productivos y ser responsable del 37,6\% del total del empleo en las Islas ${ }^{21}$, el estudio, ratifica el papel clave que desempeña el turismo en el sistema productivo canario por su capacidad de dinamización de otros sectores.

La dependencia de la economía canaria del turismo sigue en aumento, en 2016 se registró el mayor crecimiento de la actividad turística de los últimos diez años, según el informe Impactur del lobby hotelero Exceltur. El turismo genera ya el 34\% de la riqueza del archipiélago, y prácticamente el $40 \%$ del total del empleo ${ }^{22}$

Esto explica que sea precisamente ese año, 2015, en el que se apruebe el Decreto 113/2015, de 22 de mayo, por el que se aprueba el Reglamento de las viviendas vacacionales de la Comunidad Autónoma de Canarias.

El artículo 8 del Decreto 113/2015, recoge las condiciones de uso y los requisitos de seguridad, estableciendo que las personas propietarias de las viviendas, ya sean personas físicas o jurídicas encargadas de la gestión de ese alojamiento, están obligadas a remitir a la Dirección General de la Policía la información relativa a la estancia de las personas que se alojan en ella, de acuerdo con las normas legales de registro documental e información, tal y como aparece recogido en la normativa vigente de protección de la seguridad ciudadana.

La Comunidad de Canarias sí regula la obligatoriedad para este tipo de alojamientos de dar traslado a las Fuerzas y Cuerpos de Seguridad del Estado de los partes de entrada y salida de viajeros.

\subsubsection{CATALUÑA}

Cataluña es el primer destino turístico de España y uno de los principales en el mundo, con un producto diversificado y maduro.

\footnotetext{
21 ESTUDIO IMPACTUR CANARIAS 2017 EXCELTUR Disponible en: https://www.exceltur.org/wp-content/uploads/2018/07/IMPACTUR-Canarias-2017.pdf.

${ }^{22}$ CABRERA CORREA D; GONZALEZ FERNANDEZ, M, Y LUIS GONZALEZ, R; Turismo senior en Tenerife. Facultad de Economía, Empresa y Turismo, Universidad de La Laguna 2018. Disponible en:

https://riull.ull.es/xmlui/bitstream/handle/915/12055/Turismo\%20senior\%20en\%20Tenerife.pdf?s equence $=1 \&$ is Allowed $=\mathrm{y}$.
} 
Aun así, Cataluña perdió entre un 15\% y un 20\% de turistas internacionales en el tercer trimestre de 2017 debido al proceso independentista, según los cálculos de la Organización Mundial del Turismo (OMT) ${ }^{23}$.

Además de la consulta independentista, Cataluña se enfrenta a otros problemas como el auge del fenómeno conocido como turismofobia, que ha supuesto un freno al sector turístico en Barcelona.

La Orden IRP / 418/2010, de 5 de agosto, sobre la obligación de registro y comunicación a la Dirección General de la Policía de las personas que se alojan en los establecimientos de hospedaje ubicados en Cataluña IRP/418/2010, en su artículo 29 regula la identificación de las personas usuarias, estableciendo que se deberá acreditar la identidad del cliente a través de la exhibición de su documento en el momento de la entrada en el establecimiento, de identidad, también establece que dicho documento debe ser devuelto inmediatamente.

El establecimiento debe llevar el registro de las personas alojadas y remitirlo a la Dirección General de la Policía, conteniendo toda la información relativa a la estancia de las personas que se alojan, tal y como aparece recogida en la normativa de seguridad ciudadana aplicable a todos los establecimientos que facilitan, mediante precio, alojamiento a las personas.

Además de la Orden de Interior, Relaciones Institucionales y Participación 418/2010 de 5 de agosto, también es de aplicación el Decreto 159/2012, de 20 de noviembre, de establecimientos de alojamiento turístico y de viviendas de uso turístico, que en su artículo 70 relativo al registro de ocupantes establece que bien el propietario o bien el gestor del alojamiento, debe enviar a la Dirección General de la Policía la información sobre las personas que se van a alojar, para cumplir la normativa de seguridad ciudadana.

\subsubsection{CASTILLA LA MANCHA}

En Castilla la Mancha la normativa aplicable al respecto es la Ley 8/1999, de 26 de mayo, de Ordenación del Turismo de Castilla-La Mancha ${ }_{2}$ que delimita su ámbito de aplicación, tal y como aparece recogido en su art.2:" A las personas físicas o jurídicas que realicen una actividad turística en la Comunidad Autónoma de Castilla-La Mancha".

Es curioso que a lo largo de la norma no haga mención expresa a la obligatoriedad de tener un libro registro que contenga los partes de entrada de viajeros para comunicarlo a las Fuerzas y Cuerpos de Seguridad del Estado, y sin embargo el Artículo 61, tipifica como falta el incumplimiento de las disposiciones relativas a la información: "Se consideran infracciones leves las simples inobservancias de las disposiciones contenidas en la presente Ley y en la normativa respectiva de aplicación, sin trascendencia directa de carácter económico ni perjuicio grave para los usuarios, que no estén tipificadas como falta grave o muy grave, y en todo caso:

2. El incumplimiento de las disposiciones relativas a información o publicidad, libros o registros establecidos obligatoriamente por la normativa turística”.

Se trata de la única mención en su normativa al registro de clientes.

${ }^{23}$ Según los cálculos de la ORGANIZACIÓN MUNDIAL DEL TURISMO (OMT) dados a conocer por el nuevo Secretario general del organismo, Zurab Pololikashvili, en su primer acto en Madrid al frente de la institución, enero 2018. 


\subsubsection{CASTILLA Y LEON}

El Decreto 3/2017, de 16 de febrero, por el que se regulan los establecimientos de alojamiento en la modalidad de vivienda de uso turístico en la Comunidad de Castilla y León, es la normativa aplicable en la Comunidad de Castilla y León. Aquí el turismo constituye un sector estratégico, que se encuentra en constante evolución, existiendo una mayor demanda de alojamientos en la modalidad de vivienda de uso turístico.

El incremento de la demanda por los turistas de distintos tipos de establecimientos de alojamiento, pone de manifiesto la necesidad de ampliar la oferta más allá de los establecimientos turísticos recogidos expresamente en el artículo 30 de la Ley 14/2010, de 9 de diciembre, de Turismo de Castilla y León, que son establecimientos de alojamiento hotelero, de turismo rural, apartamento turístico, camping y albergue en régimen turístico.

Llama la atención que en dicho Decreto, pese a su fecha, no se haga mención al parte de entrada de viajeros.

\subsubsection{COMUNIDAD VALENCIANA}

La importancia del turismo en la Comunidad Valenciana es innegable, se sitúa por detrás de Andalucía, como segunda región con mayores ingresos por la actividad turística.

El impacto económico del turismo en la Comunidad Valenciana fue de 15.929 millones de euros el pasado año, el 14,6\% del Producto Interior Bruto (PIB) autonómico, y permitió la creación de 287.749 puestos de trabajo. Así se recoge en el informe Impactur Comunidad Valenciana 2017, elaborado por Hosteltur ${ }^{24}$.

La normativa aplicable ${ }^{25}$ en cuanto a Registro de viajeros en la Comunidad Valenciana es la Ley 15/2018, 7 de junio, de turismo, ocio y hospitalidad de la Comunitat Valenciana ${ }^{26}$ junto a la normativa específica contenida en el Decreto 92/2009, de 3 de julio, del Consell, por el que se aprueba el reglamento regulador de las viviendas turísticas denominadas apartamentos, villas, chalés, bungalows y similares, y de las empresas gestoras, personas jurídicas o físicas, dedicadas a la cesión de su uso y disfrute, en el ámbito territorial de la Comunitat Valenciana ${ }^{27}$.

${ }^{24}$ HOSTELTUR, El turismo genera 16 millones $€$ en la Comunidad Valenciana. 2018. [Consulta: 12/01/2019]. Disponible en: https://www.hosteltur.com/110284_el-turismo-genero-cerca-de-16000-m-y287749-empleos-en-2017.html

${ }^{25}$ FEDERACION EMPRESARIAL DE ASOCIACIONES TERRITORIALES DE AGENCIAS DE VIAJES ESPAÑOLAS. Disponible en: http://fetave.es/legislacion/apartamentos-turisticos.htm

${ }^{26}$ DOGV no 8313 de 08/06/2018 DIARIO OFICIAL DE LA COMUNIDAD VALENCIANA, Presidencia de la Generalitat, LEY 15/2018, 7 de junio, de la Generalitat, de turismo, ocio y hospitalidad de la Comunidad Valenciana [2018/5692] Disponible en: https://www.dogv.gva.es/datos/2018/06/08/pdf/2018_5692.pdf

${ }^{27}$ DOCV núm. 6051 de 07/07/2009 DIARIO OFICIAL DE LA COMUNIDAD VALENCIANA, Conselleria de Turismo, DECRETO 92/2009, de 3 de julio, del Consell, por el que aprueba el reglamento regulador de las viviendas turísticas denominadas apartamentos, villas, chalés, bungalows y similares, y de las empresas gestoras, personas jurídicas o físicas, dedicadas a la cesión de su uso y disfrute, en el ámbito territorial de la Comunitat Valenciana. [2009/7961] http://www.dogv.gva.es/datos/2009/07/07/pdf/2009_7961.pdf 
Tras la entrada en vigor de la Ley 15/2018, de 7 de junio, la vivienda debe disponer de licencia de ocupación o cédula de habitabilidad y debe estar suficientemente amueblada y dotada de los aparatos y enseres necesarios para su ocupación inmediata, y en perfecto estado de higiene. Los requisitos obligatorios según la categoría se encuentran en el Anexo I del Decreto 92/2009, de 3 de julio, del Consell28, entre los que se ha de acreditar que la vivienda de uso turístico dispone del informe favorable de compatibilidad urbanística emitido por el ayuntamiento del municipio en el que radica la vivienda que pretende ser de uso turístico. El informe favorable de compatibilidad ha de tener un código seguro de verificación (CSV) que permita la verificación de la integridad y autenticidad de documentos electrónicos, así como la obtención de copias electrónicas de los mismos ${ }^{29}$.

De acuerdo con la normativa del Ministerio del Interior ${ }^{30}$ debe cumplimentar un parte de entrada de viajeros, confeccionado con arreglo a un modelo oficial, y remitirlo a la correspondiente Comisaría de Policía o Puesto de la Guardia Civil, en función de la localidad donde se ubique la vivienda. La normativa aplicable se encuentra recogida en la Orden 1922/2003 de 3 de julio sobre libros-registro y partes de entrada de viajeros en alojamientos turísticos.

\subsubsection{GALICIA}

La norma fundamental es el Decreto 12/2017, de 26 de enero, por el que se establece la ordenación de apartamentos turísticos, viviendas turísticas y viviendas de uso turístico en la Comunidad Autónoma de Galicia, publicado en el DOGA el 10 de febrero de 2017, y con entrada en vigor el 10 de mayo de 2017.

En su artículo 11 determina que los apartamentos y las viviendas turísticas deberán cumplir la normativa vigente en materia de libros registro y partes de entrada de personas viajeras $^{31}$. De una manera expresa en su artículo 40.4 extiende la obligación al cumplimiento de la normativa vigente en materia de libros registro y partes de entrada de viajero a las viviendas de uso turístico.

\subsubsection{MADRID}

En el caso de Madrid, la Delegación del Gobierno lleva tiempo trabajando con la Comunidad de Madrid para establecer una colaboración más estrecha que redunde en

${ }^{28}$ DIARIO OFICIAL DE LA COMUNIDAD VALENCIANA Decreto 92/2009, de 3 de julio, del Consell, por el que aprueba el reglamento regulador de las viviendas turísticas denominadas apartamentos, villas, chalés, bungalows y similares, y de las empresas gestoras, personas jurídicas o físicas, dedicadas a la cesión de su uso y disfrute, en el ámbito territorial de la Comunidad Valenciana. http://www.dogv.gva.es/auto/dogv/docvpub/rlgv/2009/D_2009_092_ca_D_2015_075.pdf

${ }^{29}$ COMUNIDAD VALENCIANA TURISMO http://www.turisme.gva.es/opencms/opencms/turisme/es/contents/tramitacion/empresa_turistica/faq_preg untas_frecuentes_viviendas_turisticas.html

30 MINISTERIO DEL INTERIOR Partes de entrada y libros registro http://www.interior.gob.es/web/servicios-alciudadano/seguridad/libro-registro-de-viajeros/partes-deentrada-y-libro-registro

31 PARTEE, Normativa de partes de entrada de viajeros y libros-registro en alquiler turístico. Disponible en: https://partee.es/normativa-de-libros-registro-y-partes-de-entrada-de-viajeros/ 
una mayor seguridad en los apartamentos turísticos, que durante los últimos años, al igual que en otras grandes ciudades españolas, han proliferado, sobre todo en el centro de la ciudad.

La Comunidad de Madrid regula los apartamentos y viviendas de usos turísticos en el Decreto 29/2019, de 9 de abril, del Consejo de Gobierno, por el que se modifica el Decreto 79/2014, de 10 de julio, por el que se regulan los Apartamentos Turísticos y las Viviendas de Uso Turístico de la Comunidad de Madrid.

Por el contrario, el Ayuntamiento regula los apartamentos y viviendas de usos turísticos en el Plan Especial de regulación del uso de servicios terciarios en la clase de hospedaje (PEH).

El Ayuntamiento de Madrid ha votado de manera definitiva en marzo de 2019, el Plan Especial de Regulación del Uso de Hospedaje (PEH), que incorporará más restricciones a dicha modalidad. El PEH nace con el propósito de adaptar dicha normativa al Plan General de Ordenación Urbana de Madrid.

El Plan incluye como nuevas condiciones el nivel de uso del edificio en cuestión, la protección del mismo, y la zona donde esté ubicado.

Así el Ayuntamiento considera que en el caso de que la duración del arrendamiento sea superior a los 90 días por año, estaríamos ante una actividad comercial, sin embargo no sería compatible con incompatible con un uso residencial, por lo que sería preceptiva la solicitud de la correspondiente licencia, sin embargo cuando el alquiler sea inferior a 90 días por año, no se requerirá dicha licencia, por considerarse economía colaborativa. Por el contrario, el Ayuntamiento regula los apartamentos y viviendas de usos turísticos en el Plan Especial de regulación del uso de servicios terciarios en la clase de hospedaje (PEH).

El Decreto 29/2019 propone incorporar de forma específica nuevas obligaciones a los titulares o propietarios en la gestión de su actividad, con la finalidad de mejorar la calidad turística y la seguridad ciudadana, tal y como disponer de hojas de reclamación, tener contratado un seguro de responsabilidad civil o establecer la obligación a los propietarios o gestores de remitir a la Dirección General de la Policía la información relativa a los usuarios de las viviendas de uso turístico, de acuerdo a la normativa nacional en materia de protección ciudadana, así como un certificado de idoneidad ${ }^{32}$.

Remite además, en cuanto a régimen sancionador a lo dispuesto en el capítulo II, del título IV de la Ley 1/1999, de 12 de marzo, de Ordenación del Turismo de la Comunidad de Madrid, de forma que el incumplimiento de las disposiciones relativas a documentación, libros o registros establecidos obligatoriamente para el adecuado régimen y funcionamiento de la empresa, instalación o servicio y como garantía para la protección del usuario supondrá una infracción leve.

Este conjunto de nuevas obligaciones ha sido recibido con gran preocupación por parte del sector de los alojamientos turísticos hasta el punto de considerar que en la práctica puede implicar una prohibición encubierta de su actividad. Por otra parte, no se puede obviar que este tipo de limitaciones afectarán en mayor medida a los propietarios de una única vivienda o a los que comercializan en períodos concretos la suya propia que a las grandes empresas propietarias de múltiples alojamientos, con mayor capacidad de gestión de su negocio. Finalmente, por tanto, lo que se eliminaría sería la práctica más intrínsecamente ligada a la esencia y concepto originario de la economía colaborativa.

\footnotetext{
${ }^{32}$ GAMELLA, S. 2019 ¿El fin de las viviendas turísticas en Madrid? Editorial Sepin.
} 


\subsubsection{MURCIA}

En el caso de la Región de Murcia resulta de aplicación el Decreto número $75 / 2005$, de 24 de junio, por el que se regulan los apartamentos turísticos y alojamientos vacacionales en dicho Decreto se hace mención expresa a la obligatoriedad de inscribir en el libro registro de clientes a los usuarios, tal y como se recoge en su artículo 26:

“1. Será requisito indispensable para ocupar el alojamiento, la previa inscripción de los usuarios en el correspondiente Libro Registro, mediante exhibición de los documentos acreditativos de su identidad. En la inscripción se hará constar nombre y apellidos de todos los usuarios y la fecha de entrada y salida”.

\subsubsection{PAIS VASCO}

La normativa de turismo se encuentra recogida en la Ley 13/2016, de 28 de julio, de Turismo, donde solo se hace mención de dicho registro en el art.93 dedicado a las Infracciones leves:

"10. El incumplimiento de las obligaciones formales establecidas por la legislación turística relativas a documentación, libros o registros, así como la no conservación de la documentación obligatoria durante el tiempo establecido reglamentariamente".

La Orden de 18 de mayo de 2012, del Consejero de Interior regula la forma de cumplimentar las obligaciones de registro documental e información a la Ertzaintza relativa a la estancia de personas alojadas en establecimientos turísticos ${ }^{33}$.

\subsubsection{BALEARES}

La normativa aplicable está constituida por la Ley 8/2012, de 19 de julio del turismo de las Illes Balears. B.O.I.B. núm. 106, de 21 de julio de 2012, derogada en parte y más específicamente por la Ley 6/2017, de 31 de julio, de modificación de la Ley 8/2012, de 19 de julio, del turismo de las Illes Balears, relativa a la comercialización de estancias turísticas en viviendas. B.O.I.B. núm. 93 de 31 de julio de 2017.

En el caso del alquiler en Formentera, existen unos requisitos específicos recogidos en el Reglamento 1/2015, del Consejo Insular de Formentera, por el que se regula la comercialización de estancias turísticas en viviendas en la isla de Formentera, B.O.I.B núm. 60, 23 de abril de 2015, y en el Plan de calidad de Formentera, del Consejo Insular de Formentera, para las viviendas objeto de comercialización turística para el periodo 2015-2019, de Formentera, B.O.I.B núm.60, 23 de abril de 2015.

Entre los requisitos que se deben cumplir en materia de control policial en Islas Baleares para el alquiler de viviendas de uso turístico figuran:

\footnotetext{
${ }^{33}$ ERTZAINTZA

Registro

Hostelero.

Disponible

en:

https://www.ertzaintza.eus/wps/portal/ertzaintza/!ut/p/z0/04_Sj9CPykssy0xPLMnMz0vMAfIjo8ziPd2MP ULD3AwMDMy8zQw8fdyNLEzNDL2Cgs31g1OL9QuyHRUBvvLPfQ!!/https://www.ertzaintza.eus/wp s/portal/ertzaintza/!ut/p/z0/04_Sj9CPykssy0xPLMnMz0vMAfIjo8ziPd2MPULD3AwMDMy8zQw8fdyN LEzNDL2Cgs31g1OL9QuyHRUBvvLPfQ!!/
} 
1. Registro de entrada de los turistas. Requiere el envío a la Dirección General de la Policía de información sobre la estancia de las personas alojadas, de conformidad con la normativa de seguridad ciudadana.

El propietario de una vivienda de uso turístico está, por tanto, obligado a remitir a la Dirección General de Policía, la información relativa a la estancia de las personas que se alojan en ellos, de acuerdo con las normas legales de registro documental e información que se exigen en la normativa vigente en materia de protección de la seguridad ciudadana y demás disposiciones aplicables ${ }^{34}$.

\section{CONCLUSIONES}

Tras el análisis realizado a la normativa de las distintas comunidades autónomas aplicable al control y registro de usuarios en los alojamientos de uso turístico, se puede comprobar, que pese a los distintos grados de desarrollo de la normativa, en función de las diferentes Comunidades Autónomas, la regulación de los alojamientos turísticos, desde el punto de vista de la seguridad, no ha constituido una prioridad para el legislador.

Si tenemos en cuenta los principios de Legalidad y Seguridad jurídica, nos daríamos cuenta de la importancia de contar con una normativa legal y homogénea en las distintas Comunidades Autónomas.

Dichos principios tan importantes para el mundo del Derecho, quedarían cubiertos si existiera una regulación que fuera homogénea en las distintas Comunidades Autónomas, abogando por el cumplimiento de los requisitos impuestos, como el registro de la actividad y las obligaciones fiscales, pero siempre desde el respeto a los principios de libertad de empresa, y con una reducción importante e incluso ausencia de las restricciones impuestas a esta modalidad alojativa. Una práctica contraria a la ocurrida en Madrid, donde a través de la normativa publicada por el Ayuntamiento de Madrid, se prohíbe la actividad de apartamentos y viviendas de uso turístico en la zona céntrica de Madrid porque está suspendida a la concesión de licencias. Y fuera de la zona centro se podrá ejercer dicha actividad con el cumplimiento tanto de la normativa autonómica como local desde el primer día de su entrada en vigor. Y en el caso de viviendas dadas de alta antes de abril del 2019, se les dará un plazo de 6 meses para adaptarse a la nueva normativa.

Esta deficiencia y necesidad regulatoria ha sido también destacada por la Alianza para la Excelencia Turística, Exceltur, quien entre las iniciativas planteadas de cara al 2019 propone: “Acelerar el proceso de implantación de la nueva y reciente regulación nacional para los pisos turísticos y su posterior control del Gobierno, con las

${ }^{34}$ Baleares: guía definitiva para el alquiler de estancias turísticas en viviendas (etv), Disponible en: https://alquilerviviendavacacional.com/baleares-guia-definitiva-alquiler-estancias-turisticas-viviendasetv/) 
CCAA, que evite las externalidades y posibles situaciones de turismofobia derivadas de su descontrolado crecimiento ${ }^{35}$ ".

También sostiene Exceltur que la normativa vigente en España para la oferta de alquiler de viviendas por parte de particulares no es uniforme, ya que las únicas coincidencias que existen son la inscripción en un registro automático y la prohibición de alquilar habitaciones sueltas

Pero no es posible afrontar este problema sin propuestas que contemplen a todas las partes implicadas, y por ello, al tiempo que se unifica la normativa y por tanto los requisitos y obligaciones, es imprescindible trabajar en la concienciación de los propios ciudadanos, que conviven con este turismo, para minimizar las situaciones de rechazo al mismo.

No podemos olvidar que las cada vez más frecuentes conductas, englobadas bajo lo que se denomina turismofobia, inciden también en la seguridad.

Se ha discutido mucho sobre si las Comunidades de propietarios debían tener potestad para poder prohibir un alquiler vacacional sin que se requiera una unanimidad, sino una mayoría cualificada, limitada, por supuesto, a estancias de corta duración en las que el inquilino hiciese un uso inadecuado del inmueble. A nuestro juicio, si debería existir dicha potestad. Es más, nuestra propuesta iría encaminada a que en la regulación del alquiler vacacional se reconociese posibilidad de que en las juntas se pudieran tomar incluso medidas judiciales, encaminadas a mejorar la convivencia en dicha Comunidad. El hecho de que en muchas ocasiones protestas de los vecinos caigan en saco roto, puede desembocar en situaciones muy desagradables de rechazo al turismo.

Esta propuesta, aunque no exenta de debate, ya se ha implantado en algunos casos, como por ejemplo en Andalucía, donde el Decreto 28/2016, de 2 febrero, recoge la expulsión inmediata del inmueble de los inquilinos que no respeten las normas de convivencia.

Pero además, hay que tener en cuenta que los vecinos pueden y deben jugar un papel activo a la hora de identificar conductas sospechas o actitudes que puedan suponer un riesgo; y su colaboración es por tanto fundamental para garantizar la seguridad.

Una sencilla y obvia medida, aunque eficaz, también incluida en esta última Comunidad Autónoma, es la entrega por parte del propietario al inquilino de las normas de comportamiento en dicha comunidad de propietarios, contemplando aspectos como el horario para sacar la basura, el horario para poner música, hacer ruido, etc. Esta herramienta es además especialmente útil si previamente se consensua con los propios vecinos.

Cuando el inquilino causa algún daño en el inmueble objeto del alquiler, el propietario es el responsable, ¿Pero qué ocurre si ese daño lo causa en las zonas comunes del edificio? Esta situación genera un conflicto que se resolvería mediante la obligatoriedad de suscripción de un seguro por parte del propietario del inmueble, que cubra ese tipo de contingencias también en las zonas comunes, no solo en su alojamiento objeto del alquiler.

35 EXCELTUR, Perspectivas turísticas: valoración turística empresarial de 2018 y perspectivas para 2019. https://www.exceltur.org/wp-content/uploads/2019/01/Informe-Perspectivas-N67-Balance-dela\%C3\%B10-2018-y-previsiones-para-2019.pdf 
En todo caso, y desde el punto de vista concreto del control de estos alojamientos, lo primero que se evidencia es la falta de uniformidad de criterios a la hora de regular los requisitos para el registro de clientes con respecto a los partes de entrada de viajeros; lo que unido a la levedad de las sanciones, en muchos casos multas que no superan los $600 €$ en su mayor gradación, ha provocado una escasa repercusión de esta normativa.

Todo ello provoca que no se haya producido todavía una incidencia clara en materia de seguridad. Y ello es así, además en una doble vertiente, ya que tampoco se detecta la falta de control como un riesgo para el turismo, que podría provocar su deslocalización en favor de otros destinos.

Aún en los casos concretos en que se ha legislado y establecido de forma clara esta obligación surge el problema de la inexistencia de mecanismos de control y de medios por parte de los poderes públicos que permitan ejercer un control de su cumplimento. Estas viviendas escapan al control de la administración al no poderse ni siquiera cuantificar, por lo que la supervisión efectiva de las mismas es prácticamente imposible a día de hoy.

Por otra parte, tampoco se puede obviar, que el propietario y gestor de muchos de estos alojamientos no es profesional y que no resulta viable imponerle obligaciones de difícil cumplimiento que acabarán provocando el efecto contrario.

No obstante, se puede apreciar un ligero cambio en la percepción y recepción de este tipo de obligaciones que se ha visto reflejado en la proliferación de aplicaciones como Chekin $^{36}$ o Partee ${ }^{37}$ que facilitan el proceso de recabar los datos de los huéspedes y enviarlos a los Cuerpos y Fuerzas de Seguridad del Estado, permitiendo además almacenar los partes de entrada durante tres años.

La propia Policía ha puesto en marcha un sistema de registro, denominado Webpol, que exige una solicitud de alta presencial asignando después un número de usuario y clave para entrar en el sistema ${ }^{38}$.

Queda sin embargo un largo camino por recorrer, especialmente en materia de formación y concienciación ciudadana, siendo muy conscientes de que lamentablemente, la seguridad sólo se percibe como una prioridad cuando ya es un problema.

\section{BIBLIOGRAFÍA}

Baleares: guía definitiva para el alquiler de estancias turísticas en viviendas (etv) [Consulta: 11/02/2019] Disponible en: https://alquilerviviendavacacional.com/balearesguia-definitiva-alquiler-estancias-turisticas-viviendas-etv/)

BOIX, A. Una regulación para la economía colaborativa eficiente y cuidadosa. El Diario.es 2017 [Consulta: 20/01/2019] Disponible en:

\footnotetext{
${ }^{36}$ CHECK_IN App de Registro de Viajeros Disponible en: https://www.checkinscan.com/es/appregistro- viajeros/

${ }^{37}$ PARTEE, Normativa de partes de entrada de viajeros y libros-registro en alquiler turístico. [Consulta 10/03/2019] Disponible en: https://partee.es/normativa-de-libros-registro-y-partes-de-entradade-viajeros/
}

${ }^{38}$ DIRECCION GENERAL DE LA POLICIA Comunicación de datos. D.G. P. 2017. Disponible en: www.://webpol.policia.es/e-hotel/. 
https://www.eldiario.es/cv/arguments/regulacion-economia-colaborativa-eficientecuidadosa_6_629297068.html

CABRERA CORREA D; GONZALEZ FERNANDEZ, M, Y LUIS GONZALEZ, R; Turismo senior en Tenerife. Facultad de Economía, Empresa y Turismo, Universidad de la Laguna. 2018. [Consulta: 02/03/2019] Disponible en: https://riull.ull.es/xmlui/bitstream/handle/915/12055/Turismo\%20senior\%20en\%20Ten erife.pdf?sequence $=1 \&$ isAllowed $=\mathrm{y}$

CATALUNYAPRESS, La OMT calcula que el turismo en Catalunya cayó hasta un 20\% en el tercer trimestre, enero de 2018. [Consulta: 20/11/2018]Disponible en: https://www.catalunyapress.es/texto-diario/mostrar/984327/omt-calcula-turismocatalunya-cayo-hasta-20-tercer-trimestre

COMUNIDAD VALENCIANA: TURISMO. [Consulta 01/01/2019] Disponible en:

http://www.turisme.gva.es/opencms/opencms/turisme/es/contents/tramitacion/empresa turistica/faq_preguntas_frecuentes_viviendas_turisticas.html.

CHEKIN. Cómo hacer el registro de viajeros en hospederías (guardia civil) [Consulta: 03/03/2019] Disponible en: https://chekin.io/blog/como-hacer-el-registro-deviajeros-en-hospederias-guardia-civil/

CHECK_IN App de Registro de Viajeros Consulta: [10/02/2019] Disponible en: https://www.checkinscan.com/es/app-registro- viajeros/

DIRECCION GENERAL DE LA POLICIA Comunicación de datos. D.G. P. 2017. [Consulta: 16/03/2019] Disponible en: https://webpol.policia.es/ehotel/login?error=SessionTimeout\#

DIARIO OFICIAL DE LA COMUNIDAD VALENCIANA, Presidencia de la Generalitat, LEY 15/2018, 7 de junio, de la Generalitat, de turismo, ocio y hospitalidad de la Comunidad Valenciana [2018/5692].

DIARIO OFICIAL DE LA COMUNIDAD VALENCIANA, Conselleria de Turismo, DECRETO 92/2009, de 3 de julio, del Consell, por el que aprueba el reglamento regulador de las viviendas turísticas denominadas apartamentos, villas, chalés, bungalows y similares, y de las empresas gestoras, personas jurídicas o físicas, dedicadas a la cesión de su uso y disfrute, en el ámbito territorial de la Comunitat Valenciana. [2009/7961]

DIARIO OFICIAL DE LA COMUNIDAD VALENCIANA Decreto 92/2009, de 3 de julio, del Consell, por el que aprueba el reglamento regulador de las viviendas turísticas denominadas apartamentos, villas, chalés, bungalows y similares, y de las empresas gestoras, personas jurídicas o físicas, dedicadas a la cesión de su uso y disfrute, en el ámbito territorial de la Comunidad Valenciana. http://www.dogv.gva.es/auto/dogv/docvpub/rlgv/2009/D_2009_092_ca_D_2015_075.p $\underline{\mathrm{df}}$

ELMUNDO, Becares, R. Control policial a los pisos turísticos, 2017 [Consulta: 10/10/2018] Disponible en: http://www.elmundo.es/madrid/2017/11/20/5a0f1a80e5fdeae93b8b457a.html

ELMUNDO, Colmenero, R. Más de 125.000 plazas turísticas escapan al control antiterrorista. 2016. [Consulta: 10/02/2019] Disponible en: http://www.elmundo.es/baleares/2016/10/17/58047169268e3ec9198b45f4.html

ERTZAINTZA Registro Hostelero. [Consulta: 22/01/2019]Disponible en: https://www.ertzaintza.eus/wps/portal/ertzaintza/!ut/p/z0/04_Sj9CPykssy0xPLMnMz0v 
MAfIjo8ziPd2MPULD3AwMDMy8zQw8fdyNLEzNDL2Cgs31g1OL9QuyHRUBvvL PfQ!!/

EXCELTUR, Alojamientos turísticos en viviendas de alquiler: Impactos y retos asociados. Junio. 2015 [Consulta: 02/04/2018] Disponible en: http://www.exceltur.org/wp-content/uploads/2015/06/Alojamiento-tur\%C3\%ADsticoen-viviendas-de-alquiler-Impactos-y-retos-asociados.-Informe-completo.-Exceltur.pdf

EXCELTUR, Urbantur. Monitor de Competitividad Turística de Ciudades. 2016. [Consulta: 12/03/2019] Disponible en: https://www.exceltur.org/urbantur/

EXCELTUR IMPACTUR CANARIAS, Exceltur con el Gobierno de Canarias presentan el impacto económico del turismo en Canarias para el 2017. [Consulta: 24/11/2018] Disponible en: https://www.exceltur.org/wpcontent/uploads/2018/07/IMPACTUR-Canarias-2017-julio-2018-.pdf

FEDERACION EMPRESARIAL DE ASOCIACIONES TERRITORIALES DE AGENCIAS DE VIAJES ESPAÑOLAS. [Consulta: 27/02/2019] Disponible en: http://fetave.es/legislacion/apartamentos-turisticos.htm

FERNANDEZ, N. El alojamiento colaborativo. Tirant lo Blanch. Pags. 29-30. Valencia. España. 2017.

FUNDACIÓN INNOVACIÓN BANKINTER, Modelos de negocio disruptivos.

2018. [Consulta: 20/01/2019] Disponible en: https://www.fundacionbankinter.org/documents/20183/156075/Modelos+Negocio+Disr uptivos+Informe+2.pdf/e29a4961-1e52-4cfa-9de5-cc5576fab1d2

GAMELLA, S. ¿El fin de las viviendas turísticas en Madrid? Editorial Sepin 2019.Consulta: 28/05/2019 Disponible en:_https://blog.sepin.es/2019/04/viviendasturisticas-madrid/

GARIJO RUIZ, M. La economía colaborativa en el ámbito de la vivienda: cuestiones fiscales pendientes. Revista jurídica de los Derechos Sociales, Lex Social. Vol. 2/2017 julio-diciembre. Consulta: 13/12/2018. Disponible en: https://www.upo.es/revistas/index.php/lex_social/article/viewFile/2717/2178

GENERSALITAT DE CATALUNYA Registro de Turismo de Cataluña.

Establecimientos hoteleros. Consulta: 12/02/2019 Disponible en:https://web.gencat.cat/es/tramits/tramits-temes/Inscripcio-dEstabliments-Hotelers-alRegistre-de-Turisme-de-Catalunya

GONZÁLEZ CABRERA, I. Economía colaborativa: del intercambio entre pares a una nueva economía de mercado. (Un ámbito con más preguntas que respuestas). Revista de Derecho Mercantil. no. 309, 2018

HOSTELERIA MADRID, Casal, M. Madrid, la comunidad que lidera el crecimiento turístico en 2017. [Consulta: 02/12/2018] 2017 Disponible en: http://www.hosteleriamadrid.com/blog/madrid-la-comunidad-que-lidera-el-crecimientoturistico-en-2017/

HOSTELTUR, El turismo genera 16 millones $€$ en la Comunidad Valenciana. 2018. [Consulta: 12/01/2019]. Disponible en:_https://www.hosteltur.com/110284_elturismo-genero-cerca-de-16000-m-y-287749-empleos-en-2017.html

HOSTELTUR Conclusiones del Sharing Madrid sobre economía colaborativa y turismo 2017 [Consulta: 01/01/2019] Disponible en: https://www.hosteltur.com/122598_conclusiones-sharing-madrid-economiacolaborativa-turismo.html 
HOSTELTUR. Partes de entrada y libros registro de viajeros, ¿Cómo se cumplimentan? [Consulta: 05/03/2019] Disponible en: https://www.hosteltur.com/comunidad/005255_partes-de-entrada-y-libro-registro-deviajeros-como-se-cumplimentan.html

MAGRO SERVET, V. 2018 Sobre la necesaria unificación en la regulación legal del alquiler vacacional y normativa autonómica. 2018 EIDerecho.com Lefebvre. Consulta: 26/05/2019 Disponible en: https://elderecho.com/sobre-la-necesariaunificacion-en-la-regulacion-legal-del-alquiler-vacacional-y-normativa-autonomica

MARTÍN, MORAL, Ma . F. Economía colaborativa y protección del consumidor. En: Revista de Estudios Europeos. Julio-diciembre 2017,

MINISTERIO DE INDUSTRIA, ENERGÍA Y TURISMO, Plan Nacional Integral de Turismo, 2012, Secretaria de Estado de Turismo. [Consulta: 10/03/2019] Disponible en: https://turismo.gob.es/esES/PNIT/Documents/Plan\%20Nacional\%20e\%20Integral\%20d e\%20Turismo\%20(PNIT)\%202012-2015.pdf

MINISTERIO DEL INTERIOR, Plan de turismo seguro.

[Consulta: 16/03/2019] Disponible en: http://www.interior.gob.es/web/serviciosal-ciudadano/planes-de-prevencion/plan-turismo-seguro

MONTERO, J. La regulación de la economía colaborativa: Airbnb, Blablacar, Uber y otras plataformas. Tirant lo Blanch. Valencia, España, 2017.

ORTOLÁ DOMENECH, S., Novedades en la regulación de la economía colaborativa 2018 [Consulta:19/12/2018] Disponible en : https://www.auren.com/esES/blog/turismo/2018-01-18/novedades-en-la-regulacion-de-la-economia-colaborativa

OSTELEA SCHOOL OF TOURISM \& HOSPITALITY, realizado en colaboración con EAE Business School, la Universidad de Lleida y la Rey Juan Carlos. [Consulta: 20 de febrero de 2019] Disponible en: https://www.tecnohotelnews.com/2018/04/economia-colaborativa-despegue-definitivo/

PARTEE, Normativa de partes de entrada de viajeros y libros-registro en alquiler turístico. [Consulta 10/03/2019] Disponible en: https://partee.es/normativa-delibros-registro-y-partes-de-entrada-de-viajeros/

PÉREZ GUERRA, R., La intervención administrativa en la clasificación de los establecimientos hoteleros: estudio comparativo en el Derecho turístico español, en Documentación Administrativa, Presidencia del Gobierno: Gabinete Técnico para la Reforma Administrativa, $\mathrm{n}^{\circ}$ 259-260, 2011, págs. 315 a 354.

RIVERO YSERN, J. L., La transposición de la Directiva de Servicios a nivel Autonómico y Local, en El nuevo derecho administrativo: libro homenaje a prof. Dr. Enrique Rivero Ysern, Sánchez Blanco, A., Domínguez-Berrueta de Juan, M. A. y Rivero Ysern, J. L. (Coords.), Ratio Legis Librería Jurídica, Salamanca, 2011, págs. 369 a 406.

ROCA FERNÁNDEZ-CASTANYS, Ma . L. ¿Matando a la gallina de los huevos de oro?: Algunos apuntes sobre la regulación de las viviendas de uso turístico (especial referencia al caso Andaluz). En Revista Internacional de Derecho del Turismo (RIDETUR), vol. 1, n ${ }^{\circ}$ 2, 2017.

ROMÁN MÁRQUEZ, A. Las viviendas particulares dedicadas a la actividad de alojamiento turístico. Su exclusión de la ley de arrendamientos urbanos. Revista Internacional de Doctrina y Jurisprudencia.2014. [Consulta: 10/01/2019] Disponible en: 
https://idus.us.es/xmlui/bitstream/handle/11441/62407/LasViviendasParticularesDedica dasALaActividadDeAlo-4748546-1.pdf?sequence=1

SANCHEZ, P. La economía colaborativa y su impacto en el turismo. Conecta Turismo [Consulta: 05/01/2019] Disponible en: https://www.conectaturismo.com/todosobre-agencias-de-viajes/la-economia-colaborativa-impacto-turismo/

SANCHEZ GOMEZ-MERELO, M. Seguridad, factor clave para el turismo. [Consulta: 05/05/2005], Blog Belt Disponible en http://www.belt.es/expertos/HOME2_experto.asp?id=7285

SECRETARIA DE ESTADO DE TURISMO (SET) - Gobierno de España. Plan Nacional e Integral de Turismo, (PNIT) 2012-2015. SET. Madrid. [Consulta: 01/03/2019] Disponible en: http://www.minetad.gob.es/turismo/esES/PNIT/Paginas/que-es-PNIT.aspx

SUNYER, R. Hacia una economía ciudadana. Editorial UOC. Barcelona.2015

TAMAMES; R. La economía colaborativa y su difícil regulación Universo infinito en Republica.com. 2017 [Consulta: 22/02/2019] Disponible en: https://www.republica.com/universo-infinito/2017/07/05/la-economia-colaborativa-ysu-dificil-regulacion-y-ii/

TOP JURIDICO, (2018) Apartamentos turísticos y viviendas vacacionales Edit. Sepin [Consulta:30/12/2018]Disponible en: https://www.sepin.es/bibliotecaonline/verEdicion.asp?cod=0JP1F50XP0FF1Da1jP0FP1 I81zc0Fa1Jq0009P1Gk1Cj08f0yg1ys07v0Op0HL05v07u1zT0GB0Id01P0FQ0H41jC09 P1T00mi

VEINTEMINUTOS, Moreno, D. Los pisos de alquiler turístico siguen creciendo a falta de más regulación. 2018 [Consulta: 20/02/2019] Disponible en: https://www.20minutos.es/noticia/3521872/0/plazas-alquiler-turistico-crecimientoexceltur-regulacion/\#xtor=AD-15\&xts $=467263$ 


\section{ANEXO I}

MODELO DE PARTE DE ENTRADA DE VIAJEROS HOJA REGISTRO

(Rellenar con mayúsculas)

\section{Datos del establecimiento}

NIF: Parte $n^{\circ}$

Nombre del establecimiento:

Municipio:

Provincia:

Sello del establecimiento

\section{Datos del viajero}

Núm de documento de identidad:

Tipo de documento: (1)

Fecha expedición del documento: (5)

Primer apellido:

Segundo apellido:

Nombre:

Sexo: (2)

Fecha de nacimiento: (3)

País de nacionalidad:(4)

Fecha de entrada:(5) de de 20

\section{Firma del viajero}

La recogida y tratamiento se hará de acuerdo con la Ley Orgánica 15/1999, de 13 de diciembre, de Protección de Datos de Carácter Personal y al amparo de lo dispuesto en el artículo 12.1 de la Ley Orgánica 1/1992, de 21 de febrero, sobre Protección de la Seguridad Ciudadana.

Instrucciones de confección del impreso.

(1) Se admiten:

- Para españoles: D.N.I., pasaporte o permiso de conducir.

- Para extranjeros: pasaporte, carta o documento de identidad (para ciudadanos de la Unión Europea, Andorra, Islandia, Suiza, Noruega, Malta, Mónaco y San Marino). Permiso de residencia español en vigor para los extranjeros residentes en España.

Se cumplimentará: $\mathbf{D}=$ DNI, $\mathbf{P}=$ pasaporte, $\mathbf{C}=$ permiso de conducir, $\mathbf{I}=$ carta o documento de identidad, $\mathbf{N}=$ Permiso de residencia español, $\mathbf{X}=$ Permiso de residencia de otro Estado Miembro de la Unión Europea

(2) $\mathbf{F}$ = sexo femenino, y $\mathbf{M}=$ sexo masculino.

(3) En el formato AAAAMMDD, al menos se cumplimentará el año de nacimiento. El mes y el día, si se desconocen, irán como 0000.

(4) Nombre del país de nacionalidad.

(5) En el formato AAAAMMDD. 